\title{
Review of the Impact of COVID-19 on Medical Education System
}

\section{IJCRR}

Section: Healthcare

Sci. Journal Impact

Factor: 6.1 (2018)

ICV: 90.90 (2018)

(c) (7) (3)

Copyright@IJCRR

\section{Vaibhav P. Anjankar ${ }^{1}$, Ashish P. Anjankar ${ }^{2}$, Anil J. Anjankar ${ }^{3}$}

'Associate Professor, Department of Anatomy, Jawaharlal Nehru Medical College, Datta Meghe Institute of Medical Sciences DMIMS (Deemed to be University), Sawangi (Meghe), Wardha, MS, India; ${ }^{2}$ Associate Professor, Department of Biochemistry, Jawaharlal Nehru Medical College, Datta Meghe Institute of Medical Sciences DMIMS (Deemed to be University), Sawangi (Meghe), Wardha, MS, India; 3Professor, Department of Forensic Medicine and Toxicology, Jawaharlal Nehru Medical College, Datta Meghe Institute of Medical Sciences DMIMS (Deemed to be University), Sawangi (Meghe), Wardha, MS, India.

\section{ABSTRACT}

With the onset of novel coronavirus disease (COVID-19) in the second half of December 2019 in China and later spreading to the whole globe, this has turned into a global emergency. World Health Organization has declared it as a pandemic. The number of patients and deaths are increasing each day and doctors and nurses are witnessing high demands in every corner of the earth. However, the impact of coronavirus and consequent lockdown on medical education is still undetermined. In this censorious time, plenty of changes are visible which might bring noteworthy revolutions in the career development of many medical students. As very less is predicted regarding the long-lasting impact of COVID-19 on medical education, let us find out how the situation till now has impacted medical education.

The problems faced by the students and their possible solutions need to be discussed. The psycho-social impact of COVID-19 pandemic on students may lead to deterioration of their performance. In this article, the attempt is made to find out the problems faced and their solutions.

Key Words: Lockdown, Preliminary, Assessments, OSPE, Webinar, Interactive, Residency

\section{BACKGROUND}

The sunrise of the year 2020 has put the whole of the world in the new pandemic of disease by the emergence of the Coronavirus disease 2019 (COVID-19). This COVID-19 pandemic has created a global awful impact on the Economy, Health Sector, Education etc to such a level that it will take a long time for every sector to get normalized. The first case of this disease was first reported in Wuhan Province of China in late December 2019 as a cluster outbreak ${ }^{1}$. Since then, it has spread to the whole world with little or more disruption of routine life. In recent years, the world has encountered many global health emergencies like SARS in 2002-2003, Swine flu (H1N1) in 2009, EBOLA in 2016 ${ }^{1}$. But COVID-19 has affected the whole world like never before. Nobody expected such an emergency where $\%$ day modern health care systems look to be striving against this infection. Even the most developed European countries have suffered a lot due to Corona pandemic.
This Corona pandemic has affected all the sectors of the world unfavourably, affecting social, economic, financial or educational systems. Every media and other sources are talking about the impact of COVID-19 on various domains like social, psychological, financial, sports, tourism, hospitality, industries etc. however minuscule or diminutive discussion is held on the impact of COVID-19 on medical education. The virtuous and ethical medical education system is the backbone of the health care delivery system as these students are going to be responsible for the delivery of health care in the future. Already, the students and faculties are grappling with the new changes or modifications brought in the medical education system and consolidation of these changes with their plan of career development ${ }^{2}$. Changes that may seem relatively insignificant as compared to the global pandemic have the potential to be drastic turning points in the career progression of man $^{3}$. Initially, before the COVID-19 had become widespread in the world, many students had not thought much about how it was going to affect them. But

\section{Corresponding Author:}

Dr. Vaibhav Anjankar, Associate Professor of Anatomy, Jawaharlal Nehru Medical College, Datta Meghe Institute of Medical Sciences DMIMS (Deemed to be University), Sawangi (Meghe), Wardha, MS, India; Mobile: 9422856368; E-mail: vaibhav_anjankar@yahoo.co.in

ISSN: 2231-2196 (Print)

Received: 11.07 .2020
ISSN: $0975-5241$ (Online)

Revised: 03.09 .2020
Accepted: 29.09 .2020
Published: 27.10 .2020 
now, COVID-19 has disrupted all routines in hospitals, medical schools, and beyond ${ }^{2}$.

\section{How have we been affected?}

The first confirmed case of COVID-19 in India was reported on 30 January $2020^{3}$. Since then the graph of cases is sharply increasing in India. Prime Minister Narendra Damodardas Modi declared the first phase of nation wise lockdown starting from midnight of 24 March 2020 to curtail the spread of infection ${ }^{4}$. Some of the colleges had closed teaching just before the announcement of lockdown. April - May is a crucial time for medical students as most of the exam going students would be preparing for the preliminary examination. The schedule of Terminal Semester examinations has also got disturbed. Some may be expecting to complete the courses soon. Suddenly closure of classes with a complete shutdown of the college created confusion among students. All India and state counselling for PG courses were also postponed ${ }^{5}$. This led to anxiety in aspiring PG doctors dreaming for the postgraduate courses.

As per guidelines ${ }^{6}$ issued by Ministry of Health and Family Welfare, Govt. of India non-essential elective surgeries were postponed. Routine visits of patients to OPD for follow-ups were avoided or postponed ${ }^{6}$. This led to engaging residents in surgical specialities in elective and non-emergency works, as trauma and emergency cases were negligible due to lockdown. This will affect their surgical experience.

The most instantaneous effect is the complete closure of one to one lectures. These led to switching to alternative teaching approaches like recorded lectures or live streaming of lectures through a variety of apps. There are disadvantages in this system like inattentive students, connectivity problems, teaching speed of the faculty during the session, loss of one to one contact between the teacher and student. Institutions have suspended clinical demonstrations and practicals to reduce the risk of exposure to medical students ${ }^{3}$. Many prelim examinations of the students supposed to be held in May or June were either postponed or conducted.

The students sitting in the homes during lockdown are not able to discuss the topic by group discussion as many students have the habit of studying by group discussion.

Another impact of COVID-19 in the medical field is the cancellation or postponement of Medical Conferences. These conferences play a vital role in continuing medical education among the participants. The presentations that medical students give in conference or workshop are essential to building up medical student's resumes and applications for residency $^{2}$. Students will now miss this valuable experience of presentations that helped the previous generations to become future doctors.

\section{How to overcome these issues?}

University Grant Commission (UGC) released guidelines on examination and academic calendar because of COVID-19 pandemic and subsequent lockdown. It mentions that it is joint culpability of all the shareholders to manage multiple key issues relating to academic activities in the institutions. While it is important to follow the instructions and regulations announced by the Government to contain the spread of COVID-19, it is also important to continue the educational process making effective use of technology and other available options ${ }^{7}$. UGC and MHRD have emphasized to continue with the teaching-learning activities using online modes such as Google Classroom, Google Hangout, Cisco Webex Meeting, YouTube Streaming, OERs, SWAYAM Platform and SWAYAMPRABHA (available on Doordarshan (Free dish) and Dish TV), etc. The medical colleges, hospitals and universities are observing comprehensive changes. Online equivalents are now the obvious and significant necessity in the replacement of face to face classes.

University Grant Commission (UGC) ${ }^{7}$ have advised adopting alternative and simplified modes and methods of examinations like MCQ/ OMR based examinations, Open Book Examination, Open Choices, assignment/ presentation-based assessments etc. UGC also advised developing virtual classroom and video conferencing facility and training of all teachers to make use of the technology. They further suggested preparing e-content/e-lab experiments and uploading the same on their websites. Maharashtra University of Health Sciences (MUHS), Nashik confirmed that students will not be promoted to the next level without examinations ${ }^{8}$. Some universities cancelled the semester exams. But prelim exams are critical as internal assessment depends on them.

With the cancelling of lectures in the institutions, many attempts were made by the authorities to rectify the backlog. In our institutions, we started to have online classes on various app-based platforms. The recorded lectures were also provided to the students. Video recordings of Objective Structured Practical Examinations (OSPE) were also given to the students to study the practical aspects. Moreover, we conducted the dissections on the online format to correlate the practical aspects with theory classes. These alternative teaching approaches like recorded lectures or live streaming of lectures may be beneficial to students but it may have some of its inherent limitations.

Cancellation of Conferences and thus many students have lost the opportunity for personal development through conference presentations ${ }^{2}$. Use of technology to overcome these issues is the innovative idea. Webinars or online conferences are new alternatives for such cancellations. So there is a growing demand for organizing online conferences or webinars. The younger generations of students and faculties are perhaps best equipped to amalgamate technology 
and webinars into health care delivery and sharing medical knowledge in online settings ${ }^{2}$.

\section{Role of Medical Educator}

Such a crisis like COVID-19 pandemic can be seen as opportunities for medical educators to leverage technology for both undergraduate and postgraduate medical education. Traditional teaching in lecture halls or demonstrations using chalk and board is getting replaced by new online teaching methods using various app-based concepts or recorded lectures. Considering the high infectivity of coronavirus, face to face lectures in the large setting is not advisable. To overcome this, the use of technology like videoconferencing and e-learning platforms will be useful for lectures or tutorials remotely via handheld devices and laptops ${ }^{9}$. Teleconferencing can also be used to demonstrate medical procedures and surgical techniques ${ }^{10}$. The success of online teaching will be improved if students pay attention to these online platforms of teaching. To achieve this, students should be encouraged to use online resources to facilitate their learning. But this will improve individual skills only. Other attributes like teamwork, empathy, courage, and compassion are important that should be inculcated in every medical student and resident in training 9 .

The medical educator or faculties have to be more attentive and creative while taking online classes because the students who are miles away has to be watched for their continuous attention and feedback need to be taken for improvement in e-lectures. The interest of students also needs to be maintained. They should be motivated. Regardless of the unique nuances of each medical student's situation, every student will face some difficulties that have arisen due to the widespread effects of the COVID-19 pandemic ${ }^{2}$. The effect of COVID-19 has started to make an impact on other aspects of students' career progression and lives ${ }^{2}$.

A new introduction in the medical education system is Blended learning ${ }^{11,12}$ which is defined as the combination of conventional face-to-face learning and asynchronous or synchronous e-learning, has grown quickly and is now extensively used in medical education ${ }^{11,12}$. Another alternative to enhance active learning is Flipped classrooms where students are first exposed to particular topic via online or offline available resources. Subsequently face-to-face class time can then be devoted to student-centered activities ${ }^{11,13}$. The current day medical education is highly enlightened by recent online accessible technological improvements in simulation and material sciences like virtual interactive anatomy dissection table, and 3D reconstruction models ${ }^{11}$.

\section{CONCLUSION}

Corona pandemic affected the medical education system in the midst of academic calendar. Two months has been passed after suspension of classes and several points needs to be clarified by authorities like exams, reopening of regular classes and clinics. Now it's time to decide the priorities and take appropriate actions. We have to learn from our experience and practical approach is to be implemented. Reflection and evaluation must follow ${ }^{13}$. Corona may show massive impact on medical education if appropriate action is not taken at the right time.

In the future, when corona will be under control, we have to rethink on medical teaching by implementing different teaching techniques complementary to conventional face-to face education.

\section{ACKNOWLEDGMENT}

Authors acknowledge the immense help received from the scholars whose articles are cited and included in references to this manuscript. The authors are also grateful to authors / editors / publishers of all those articles, journals, and books from which the literature for this article has been reviewed and discussed.

\section{Conflict of Interest: Nil}

\section{Source of Funding: Nil}

\section{REFERENCES}

1. Kannuchamy KS and Kesavelu D. Overview and Preventive Strategies of Coronavirus among Healthcare Communities. International Journal of Current Research and Review. August 2020; 12(15): 2-6. DOI: http://dx.doi.org/10.31782/ IJCRR.2020.12155

2. Ferrel MN and Ryan JJ. The Impact of COVID-19 on Medical Education. Cureus. March 31, 2020: 12(3): e7492. DOI 10.7759/cureus. 7492

3. COVID-19 Pandemic in India. [Accessed online 2020 May 26]. Available at: http: https://en.wikipedia.org/wiki/COVID-19 pandemic_in_India

4. India will be under complete lockdown for 21 days: Narendra Modi. [Accessed online 2020 May 26] Available at: https://economictimes.indiatimes.com/news/politicsand-nation/india-will-be-under-complete-lockdownstarting-midnight-narendra modi/articleshow/74796908. cms?utm_source $=$ contentofinterest\&utm_medium=text\&utm campaign $=$ cppst

5. Coronavirus Update: Medical Council of India Issues Advisory on Suspension of MBBS Classes. [Online cited on 2020 May 26] Available at: https://medicaldialogues.in/news/health/mci/coronavirus-update-medical-council-of-india-issues-advisory-on-suspensionof-mbbs-classes-64231? infinitescroll $=1$

6. Advisory for Hospitals and Medical Education Institutions by $\mathrm{MoH}$ and FW. [Accessed online 2020 May 26] Available at: https://www.mohfw.gov.in/pdf/AdvisoryforHospitalsandMedicalInstitutions.pdf

7. UGC Guidelines on Examinations and Academic Calendar for the Universities in View of COVID-19 Pandemic and Subse- 
quent Lockdown. [Accessed online 2020 May 26] Available at: https://www.ugc.ac.in/pdfnews/4276446_UGC-Guidelines-onExaminations-and-Academic-Calendar.p $\overline{d f}$

8. Maharashtra: No promotion for medical students without exams. [Accessed online 2020 May 26] Available at: http://timesofindia.indiatimes.com/articleshow/75652524. cms?utm_source $=$ contentofinterest\&utm_medium $=$ text\&utm campaign $=$ cppst

9. Zhen Chang Liang et al. Pandemics and Their Impact on Medical Training: Lessons from Singapore. Acad Med. XXXX;XX:00-00. DOI: 10.1097/ACM.0000000000003441.

10. Lamba P. Teleconferencing in medical education: A useful tool. Australas Med J. 2011;4:442-7.

11. Moszkowicz D, Duboc H, Dubertret C, Roux D, Bretagnol F. Daily medical education for confined students during COV-
ID-19 pandemic: A simple videoconference solution. Clin Anat. 2020;1-2. https://doi.org/10.1002/ca.23601

12. Vodovar, D., Ricard, J. D., Zafrani, L., Weiss, E., Desrentes, E., \& Roux, D. (2020). Assessment of newly-implemented blended teaching of intensive care and emergency medicine at Paris-Diderot University. La Revue de Médecine Interne PII: S0248-8663(20)30009-6. https://doi.org/10.1016/j. revmed.2019.12.021

13. Ramnanan, C. J., \& Pound, L. D. (2017). Advances in medical education and practice: Student perceptions of the flipped classroom. Advances in Medical Education and Practice, 13(8), 63-73.

14. Suzanne Rose. Medical Student Education in the Time of COVID-19. JAMA Published online March 31, 2020. E1-E2. 